\title{
COMMON HYPERBOLIC BASES FOR CHAINS OF ALTERNATING OR QUADRATIC LATTICES
}

\author{
RAINER SCHULZE-PILLOT
}

\begin{abstract}
We give a short and purely bilinear proof of the fact that two chains of $p$-elementary lattices with quadratic form or alternating bilinear form over $\mathbb{Z}_{p}$ or more generally over a complete discrete valuation ring have common hyperbolic bases. This fact, which is useful for the study of Bruhat-Tits buildings, has been proven before with different methods by Abramenko and Nebe and by Frisch.
\end{abstract}

\section{INTRODUCTION.}

Let $R$ be a complete discrete valuation ring with field of fractions $F, p$ a prime element in $R$. Let $V$ be a finite dimensional vector space over $F$ with a non degenerate alternating or symmetric bilinear form $b$. In the symmetric case we assume $b$ to be associated to a quadratic form $Q$ on $V$ satisfying $b(x, y)=Q(x+$ $y)-Q(x)-Q(y)$, we have then $b(x, x)=2 Q(x)$ for all $x \in V$. In the alternating case we set $Q \equiv 0$. A vector $x \in V$ is called isotropic if $x \neq 0, Q(x)=0$, a nonzero subspace or $R$-submodule $X$ is called totally isotropic if $Q(x)=b(x, y)=0$ holds for all $x, y \in X$, it is called anisotropic if $Q(x) \neq 0$ for all $x \in X \backslash\{0\}$. An $R$-lattice $\Lambda$ of maximal rank on $V$ is called $p^{r}$-maximal if it is maximal among the lattices satisfying $Q(\Lambda) \subseteq p^{r} R$ and $b(\Lambda, \Lambda) \subseteq p^{r} R$, for $r=0$ we say that the lattice is maximal. If $(V, Q)$ is anisotropic there is a unique $p^{r}$-maximal lattice on $V$, namely $\left\{x \in V \mid Q(x) \in p^{r} R\right\}$, see [6, 16.1].

It is well known (see e.g. [7, [3] for the symmetric case) that any two $p^{r_{1}}$ resp. $p^{r_{2}}$ maximal lattices $\Lambda_{1}, \Lambda_{2}$ have a common hyperbolic basis, i.e., there are vectors $\left\{e_{i}, f_{i}\right\}$ of $V$ with $b\left(e_{i}, e_{j}\right)=b\left(f_{i}, f_{j}\right)=0, b\left(e_{i}, f_{j}\right)=\delta_{i j}$ such that suitable multiples of the $e_{i}, f_{i}$ together with a basis of a maximal lattice on an anisotropic kernel of $V$ form bases of $\Lambda_{1}$ and $\Lambda_{2}$. As explained in [5], in the theory of Bruhat-Tits buildings one needs the even sharper statement that such common bases exist for pairs of certain chains of lattices. Proofs of such statements have been given in 1 using the theory of hereditary orders and in [4] using the concept of $p$-adic norms from 2. The purpose of this note is to give a short elementary proof of this fact using only the quadratic and bilinear forms. It is easy to generalize our argument to the case of hermitian forms, we leave the details of this to the reader.

\section{LATTICE CHAins}

Definition 2.1. Let $\Lambda$ be a lattice on $V$ (or a subset of a lattice spanning $V$ ). The dual lattice of $\Lambda$ is $\Lambda^{\#}:=\{x \in V \mid b(x, \Lambda) \subseteq R\}$. The lattice is called p-elementary if $\Lambda^{\#} \supseteq \Lambda \supseteq p \Lambda^{\#}$ holds. It is called $p^{r}$-modular if $\Lambda=p^{r} \Lambda^{\#}$ holds.

To appear in Mathematische Annalen, DOI: 10.1007/s00208-019-01824-5. The final publication is available at Springer via https://link.springer.com/journal/208. 
Remark. It is well known that $\Lambda^{\#}$ is a lattice on $V$ and that $\left(\Lambda^{\#}\right)^{\#}=\Lambda$ holds. The lattice is p-elementary if and only if $p R \subseteq b(x, \Lambda) \subseteq R$ holds for all primitive vectors $x \in \Lambda$.

We need a few preparations for the case of a symmetric bilinear form.

Definition 2.2. Let $(V, Q)$ be a regular quadratic space over $F$ and $\Lambda$ a lattice on $V$.

a) $\Lambda$ is called integral if $b(\Lambda, \Lambda) \subseteq R$, even if $Q(\Lambda) \subseteq R$, totally even if it is integral and $Q(x) \in b(x, \Lambda)$ holds for all $x \in \Lambda$.

b) We say that the lattice $\Lambda$ is almost p-elementary totally even (or of $p$ elementary totally even type) if $V$ has a Witt decomposition $V=V^{\text {hyp }} \perp$ $V^{\text {an }}$, where $V^{\text {hyp }}$ is a sum of hyperbolic planes and $V^{\text {an }}$ is anisotropic, such that $\Lambda=\Lambda \cap V^{\text {hyp }} \perp \Lambda \cap V^{\text {an }}$ with $\Lambda^{\text {hyp }}:=\Lambda \cap V^{\text {hyp }}$ totally even and p-elementary and $\Lambda^{\text {an }}:=\Lambda \cap V^{\text {an }}=\left\{x \in V^{\text {an }} \mid Q(x) \in R\right\}$ the unique $R$-maximal lattice on $V^{\text {an }}$.

c) If $\Lambda$ is almost p-elementary totally even, the modified dual $\Lambda^{*}$ is $\left\{x \in \Lambda^{\#} \mid\right.$ $p Q(x) \in R\}$ with the quadratic form $Q^{*}:=p Q$ and the bilinear form $b^{*}:=p b$.

Remark. a) Part c) of this definition is a slight modification of the one given by Frisch 4, who also proved a version of the next two lemmata.

b) In particular, a maximal lattice on $V$ is almost p-elementary totally even.

c) If we set $Q=0$ for an alternating bilinear form $b$, the definition of almost p-elementary totally even above coincides with p-elementary. We will use "almost p-elementary totally even" for both types of $b$ in what follows.

We also use some more terminology from the symmetric case in the alternating case as well. In particular we generalize the notion of dual lattice and $p^{r}$-modular lattice to the alternating case in the obvious way and call a lattice $R x+R y$ with $Q(x)=Q(y)=0, b(x, y)=p^{r}$ a $p^{r}$-modular hyperbolic plane.

Lemma 2.3. The modified dual $\left(\Lambda^{*}, Q^{*}\right)$ of an almost $p$-elementary totally even lattice is almost $p$-elementary totally even and one has

$$
\left((\Lambda, Q)^{*}\right)^{*}=\left(p^{-1} \Lambda, p^{2} Q\right) \cong(\Lambda, Q) .
$$

Proof. We write $\Lambda=\Lambda^{\text {hyp }} \perp \Lambda^{\text {an }}$ and have $\Lambda^{\#}=\left(\Lambda^{\text {hyp }}\right)^{\#} \perp\left(\Lambda^{\text {an }}\right)^{\#}$ with $p Q(x) \in$ $R$ for all $x \in\left(\Lambda^{\text {hyp }}\right)^{\#}$ and therefore $\Lambda^{*}=\left(\Lambda^{\text {hyp }}\right)^{\#} \perp\left\{x \in\left(\Lambda^{\text {an }}\right)^{\#} \mid p Q(x) \in R\right\}$. Obviously, $\left(\Lambda^{\text {hyp }}\right)^{\#}$, equipped with $p Q, p b$, is $p$-elementary totally even. For the anisotropic part, the set $\left\{x \in\left(\Lambda^{\text {an }}\right)^{\#} \mid p Q(x) \in R\right\}$ is a lattice which clearly is contained in the unique maximal lattice on $\left(V^{\text {an }}, p Q\right)$. For the reverse inclusion, let $x \in V^{\text {an }}$ with $Q(x) \in p^{-1} R$. If one had $x \notin\left(\Lambda^{\text {an }}\right)^{\#}$ there would exist $y \in \Lambda^{\text {an }}$ with $b(x, y)=p^{-1}$, hence $Q(x)=-b(x, a y)$ with $a \in R$. This gives $Q(x+a y) \in R$, hence $x+a y \in \Lambda^{\text {an }} \subseteq\left(\Lambda^{\text {an }}\right)^{\#}$, which contradicts $x \notin\left(\Lambda^{\text {an }}\right)^{\#}$, and we see that the maximal lattice on $\left(V^{\text {an }}, p Q\right)$ is indeed contained in $\left\{x \in\left(\Lambda^{\text {an }}\right)^{\#} \mid p Q(x) \in R\right\}$.

Finally, the same argument shows that $\left(\Lambda^{*}, p b\right)^{*}=p^{-1} \Lambda^{\text {hyp }} \perp\left\{x \in V^{\text {an }} \mid p^{2} Q(x) \in\right.$ $R\}=p^{-1} \Lambda$ with $p^{-1} \Lambda$ equipped with $p^{2} Q$.

In what follows we will identify $\left(p^{-1} \Lambda, p^{2} Q\right)$ with $(\Lambda, Q)$ and will therefore write $\left(\Lambda^{*}\right)^{*}=\Lambda$. We will also treat both cases of $b$ (symmetric or alternating) at the same time in what follows. 
Lemma 2.4. Let $\Lambda$ be an almost p-elementary totally even lattice on $V$ and let $J \subseteq \Lambda$ be a unimodular or $p$-modular hyperbolic plane which splits $\Lambda$, i.e., $\Lambda=J \perp$ $\Lambda_{1}$. Then $\Lambda_{1}$ is almost p-elementary totally even.

Proof. By definition we have $\Lambda=\Lambda^{\prime} \perp \Lambda^{\prime \prime}$, where $V^{\text {hyp }}=V^{\prime}=F \Lambda^{\prime}$ is a sum of hyperbolic planes, $V^{\text {an }}=V^{\prime \prime}=F \Lambda^{\prime \prime}$ is anisotropic, and $\Lambda^{\prime}$ is totally even $p$ elementary on $V^{\prime}$. $\Lambda^{\prime}$ has a splitting $\Lambda^{\prime}=K_{1} \perp \cdots \perp K_{s}$ into binary lattices $K_{i}$ which are unimodular or $p$-modular hyperbolic planes. At least one of the $K_{i}$ is unimodular if and only if there exists an isotropic vector $x \in \Lambda$ with $b(x, \Lambda)=R$, in particular, if $J$ is unimodular, at least one of the $K_{i}$, say $K_{1}$, is unimodular. By [6, Folgerung 4.4] there exists $\sigma \in O(\Lambda)$ with $\sigma(J)=K_{1}$ and hence $\sigma\left(\Lambda_{1}\right)=K_{1}^{\perp}$, the latter one being almost $p$-elementary totally even. Hence $\Lambda_{1}$ is almost $p$-elementary totally even too.

If $J$ is $p$-modular, $J^{*}$ is unimodular and splits $\Lambda^{*}$. By the first case, the orthogonal complement $\Lambda_{2}$ of $J^{*}$ in $\Lambda^{*}$ is almost $p$-elementary totally even, which implies that $\Lambda_{1}=\left(\Lambda_{2}\right)^{*}$ is almost $p$-elementary totally even.

Lemma 2.5. Let $\Lambda=\Lambda^{\text {hyp }} \perp \Lambda^{\text {an }}$ be an almost p-elementary totally even lattice containing isotropic vectors.

a) If the hyperbolic part $\Lambda^{\text {hyp }}$ of $\Lambda$ is not a sum of p-modular hyperbolic planes or $\Lambda=\Lambda^{\text {hyp }}$ holds, $\Lambda$ is generated by its isotropic vectors.

b) If $\Lambda^{\text {hyp }}$ is a sum of p-modular hyperbolic planes, the isotropic vectors of $\Lambda$ generate the sublattice $\{x \in \Lambda \mid Q(x) \in p R\}$.

c) One has $\{z \in p\{x \in \Lambda \mid Q(x) \in p R\} \# \mid Q(z) \in R\} \subseteq \Lambda$.

Proof. The first two assertions follow from the fact that $\Lambda^{\text {hyp }}$ is obviously generated by its isotropic vectors and that for any vector $x \in \Lambda$ satisfying $Q(x) \in Q\left(\Lambda^{\text {hyp }}\right)$ one can find $y \in \Lambda^{\text {hyp }}$ with $Q(x+y)=0$. For the last part of the lemma the set $\{x \in \Lambda \mid Q(x) \in p R\}$ generates $\Lambda$ if $\Lambda^{\text {hyp }}$ is not a sum of $p$-modular hyperbolic planes and is equal to $\{x \in \Lambda \mid Q(x) \in p R\}=\Lambda^{\text {hyp }} \perp\left\{x \in V^{\text {an }} \mid Q(x) \in p R\right\}$ otherwise. Taking duals and multiplying by $p$ we obtain the assertion.

Definition 2.6. Let $n$ denote the Witt index of $V$, i.e., the dimension of a maximal totally isotropic subspace.

A maximal admissible lattice chain $\mathcal{L}$ in $(V, Q)$ is a chain of lattices $\Lambda^{\max }=\Lambda^{(0)} \supsetneq$ $\Lambda^{(1)} \ldots \supsetneq \Lambda^{(n)}=\Lambda^{(\mathrm{min})}$, where $\Lambda^{(0)}$ is a maximal lattice on $V, n$ is the Witt index of $V$, and each $\Lambda^{(j)}$ is almost p-elementary totally even.

Remark. The last lattice $\Lambda^{\text {min }}$ of a maximal admissible lattice chain is the orthogonal sum of p-modular hyperbolic planes and a maximal lattice on an anisotropic space, whereas in $\Lambda^{\max }$ the hyperbolic planes occurring are all unimodular. Moreover, we have $p \Lambda^{\max } \subseteq \Lambda^{\min }$.

\section{Hyperbolic BASES}

Theorem 3.1. Let $\Lambda \subseteq V$ be an almost p-elementary totally even $R$-lattice on $V$. Let $X$ be a maximal totally isotropic submodule of $\Lambda$. Then there are a basis $\left(e_{1}, \ldots, e_{n}\right)$ of $X$ and vectors $f_{1}, \ldots, f_{n} \in \Lambda$ generating a totally isotropic submodule of $\Lambda$ and satisfying $b\left(e_{i}, f_{j}\right) \in\left\{\delta_{i j}, p \delta_{i j}\right\}$ such that

$$
\Lambda=\bigoplus_{i=1}^{n} R e_{i} \oplus \bigoplus_{i=1}^{n} R f_{i} \perp K
$$


where $K$ is the unique maximal lattice on an anisotropic subspace $F K$ of $V$.

Proof. We use induction on $n=\operatorname{rk}(X)$. For $n=0$ the space $V$ is $\{0\}$ in the alternating case, $\{0\}$ or anisotropic in the symmetric case, and the assertion is trivial.

Let $n \geq 1$ and assume the assertion to be true for $\operatorname{rk}(X)<n$.

If $b(X, \Lambda)=R$ we choose $x \in X, y \in \Lambda$ with $b(x, y)=1$. Replacing $y$ by $y-Q(y) x$ if necessary we may assume $y$ to be isotropic so that $R x+R y$ is a unimodular hyperbolic plane. We can then split $\Lambda$ as $(R x+R y) \perp \Lambda^{\prime}$, with $\Lambda^{\prime} \cap X=\{z \in$ $X \mid b(z, y)=0\}$ maximal totally isotropic in $\Lambda^{\prime}$ of rank $n-1$, and the induction hypothesis implies the assertion in this case.

If $b(X, \Lambda)=p R$ we have $p^{-1} X \subseteq \Lambda^{*}$ with $b^{*}\left(p^{-1} x, \Lambda^{*}\right)=R$ for all primitive vectors $x \in X$, in particular we see that $p^{-1} X$ is primitive and hence maximal totally isotropic in $\Lambda^{*}$.

Using the first case we find a basis $\left(p^{-1} e_{1}, \ldots, p^{-1} e_{n}\right)$ of $p^{-1} X$ and vectors $f_{1}^{*}, \ldots, f_{n}^{*} \in$ $\Lambda^{*}$ generating a totally isotropic submodule of $\Lambda^{*}$ and satisfying $b\left(e_{i}, f_{j}^{*}\right) \in\left\{\delta_{i j}, p \delta_{i j}\right\}$ such that

$$
\Lambda^{*}=\bigoplus_{i=1}^{n} R p^{-1} e_{i} \oplus \bigoplus_{i=1}^{n} R f_{i}^{*} \perp K,
$$

where $K$ is the unique maximal lattice on an anisotropic subspace $F K$ of $V$. Since we have $b\left(e_{i}, \Lambda^{*}\right)=b^{*}\left(p^{-1} e_{i}, \Lambda^{*}\right)=R$ for all $i$ we see that the case $b\left(e_{i}, f_{i}^{*}\right)=p$ can not occur. We set $f_{i}=p f_{i}^{*}$ for all $i$ and obtain the assertion by taking the modified dual of both sides of the last equation.

An obvious consequence is:

Corollary 3.2. With $\Lambda, b, Q$ as in the proposition all maximal totally isotropic submodules of $\Lambda$ are in the same orbit under the action of the isometry group of $(\Lambda, b, Q)$.

In particular, in the alternating case the symplectic group of $(\Lambda, b)$ (also called a local paramodular group of level $p$ if $\Lambda$ has both unimodular and $p$-modular components ) acts transitively on the set of maximal totally isotropic submodules, a fact which is well known for the integral symplectic group.

Theorem 3.3. Let $\Lambda_{\nu}^{\max }=\Lambda_{\nu}^{(0)} \supseteq \cdots \supseteq \Lambda_{\nu}^{(n)}=\Lambda_{\nu}^{\min }$ for $\nu=1,2$ be two maximal admissible lattice chains on $V$.

Then there exist isotropic vectors $e_{1}, \ldots, e_{n}, f_{1}, \ldots, f_{n} \in \Lambda_{1}^{(0)}$ with $b\left(e_{i}, e_{j}\right)=0=$ $b\left(f_{i}, f_{j}\right), b\left(e_{i}, f_{j}\right)=\delta_{i j}$ such that

$$
\Lambda_{1}^{(0)}=\bigoplus_{i=1}^{n} R e_{i} \oplus \bigoplus_{i=1}^{n} R f_{i} \perp K
$$

where $K$ is the unique maximal lattice on the anisotropic orthogonal complement of the space generated by the $e_{i}, f_{i}$, and such that

$$
\Lambda_{\nu}^{(j)}=\bigoplus_{i=1}^{n} R p^{r_{i}^{(\nu, j)}} e_{i} \oplus \bigoplus_{i=1}^{n} R p^{s_{i}^{(\nu, j)}} f_{i} \perp K
$$

holds with certain integers $r_{i}^{(\nu, j)}, s_{i}^{(\nu, j)}$ for $\nu=1,2$ and $0 \leq j \leq n$. 
Proof. We prove the assertion by induction on the Witt index $n$ of $V$. The case $n=0$ is trivial, so we assume for the rest of the argument $n>0$. In that case we notice first that $\Lambda_{\nu}^{\max }$ is generated by its isotropic vectors by Lemma 2.5 and that $b\left(x, \Lambda_{\nu}^{\max }\right)=R$ holds for each primitive isotropic vector $x \in \Lambda_{\nu}^{\max }$. On the other hand, $b\left(x, \Lambda_{\nu}^{\min }\right)=p R$ holds for all primitive isotropic $x \in \Lambda_{\nu}^{\min }$.

There exists $r \in \mathbb{N}$ with $p^{r} \Lambda_{1}^{\max } \subseteq \Lambda_{2}^{\min }, p^{r} \Lambda_{2}^{\max } \subseteq \Lambda_{1}^{\min }$, without loss of generality we may assume $p^{r-1} \Lambda_{1}^{\max } \not \subset \Lambda_{2}^{\min }$. Since $\Lambda_{1}^{\max }$ is generated by isotropic vectors we find $x \in \Lambda_{1}^{\max }$ isotropic such that $p^{r} x$ is primitive in $\Lambda_{2}^{\min }$. As noticed above we must have $b\left(p^{r} x, \Lambda_{2}^{\min }\right)=p R$.

Assume that $x$ can be chosen such that $p^{r} x$ is primitive in $\Lambda_{2}^{\max }$. One has then $b\left(p^{r} x, \Lambda_{2}^{\max }\right)=R$, and there is $0 \leq j<n$ with $b\left(p^{r} x, \Lambda_{2}^{(j)}\right)=R, b\left(p^{r} x, \Lambda_{2}^{(j+1)}\right)=$ $p R$. We choose then $x$ with $p^{r} x$ primitive in $\Lambda_{2}^{\max }$ such that the largest integer $k$ with $x \in \Lambda_{1}^{(k)}$ is as large as possible.

Choose $y \in \Lambda_{2}^{(j)}$ with $b\left(p^{r} x, y\right)=1$, we have then $p y$ primitive in $\Lambda_{2}^{(j+1)}$ and $p y \in \Lambda_{2}^{\min }$, moreover $b\left(p y, \Lambda_{2}^{\max }\right)=p R$ and hence $b\left(R p^{r} x+R p y, \Lambda_{2}^{(i)}\right)=p R$ for all $i>j$. This implies that $R p^{r} x+R p y$ splits off orthogonally in $\Lambda_{2}^{(j+1)}, \ldots, \Lambda_{2}^{\min }$, whereas $R p^{r} x+R y$ splits off orthogonally in $\Lambda_{2}^{\max }, \ldots, \Lambda_{2}^{(j)}$.

If $x$ could be chosen to be in $\Lambda_{1}^{\text {min }}$ we can split off $R x+R p^{r} y$ orthogonally in all $\Lambda_{1}^{(i)}$ and proceed by induction on $n$. Otherwise there is $0 \leq k<n$ with $x \in \Lambda_{1}^{(k)}$ and $p x$ primitive in $\Lambda_{1}^{(k+1)}$. With $y$ as above the unimodular hyperbolic plane $R x+R p^{r} y$ splits off orthogonally in $\Lambda_{1}^{\max }, \ldots, \Lambda_{1}^{(k)}$. If $b\left(p^{r} y, \Lambda_{1}^{(k+1)}\right)=p R$ holds, $R p x+R p^{r} y$ splits off orthogonally in $\Lambda_{1}^{(k+1)}, \ldots, \Lambda_{1}^{\min }$, and we can proceed by induction. Otherwise there exists $x^{\prime} \in \Lambda_{1}^{(k+1)}$ with $b\left(p^{r} x^{\prime}, \Lambda_{2}^{\max }\right)=R$, hence $p^{r} x^{\prime}$ primitive in $\Lambda_{2}^{\max }$, which contradicts our choice of $x$, so this situation can not occur and we are done with the case that $p^{r} x$ can be chosen to be primitive in $\Lambda_{2}^{\max }$.

We are left with the case that $p^{r} x$ can not be chosen to be primitive in $\Lambda_{2}^{\max }$, hence $p^{r-1} x \in \Lambda_{2}^{\max }$ for all isotropic $x \in \Lambda_{1}^{\max }$, which implies $p^{r-1} \Lambda_{1}^{\max } \subseteq \Lambda_{2}^{\max }$. If one has $p^{r-1} \Lambda_{2}^{\max } \not \subset \Lambda_{1}^{\max }$ we may interchange the two chains and reduce to the previous case, so we may assume that $p^{r-1} \Lambda_{2}^{\max } \subseteq \Lambda_{1}^{\max }$ holds as well.

Since $p^{r-1} \Lambda_{1}^{\max } \not \subset \Lambda_{2}^{\min }$ holds by assumption and $p^{r-2} x \in \Lambda_{2}^{\max }$ implies $p^{r-1} x \in$ $\Lambda_{2}^{\min }$ we can choose $x \in \Lambda_{1}^{\max }$ isotropic with $p^{r-1} x$ primitive in $\Lambda_{2}^{\max }$. Among such $x$ we choose one for which the largest integer $k$ with $x \in \Lambda_{1}^{(k)}$ is maximal. If we have $k=n$ there exists $0 \leq i<n$ such that we can choose $x \in p\left(\Lambda_{1}^{(i+1)}\right)^{*} \subseteq \Lambda_{1}^{\min }$ and we impose the additional condition that the least integer $i$ with $x \in p\left(\Lambda_{1}^{(i+1)}\right)^{*}$ is minimal. We fix these integers $i, k$ for the rest of the proof.

We notice that if $k<n$ holds for the maximal $k$ above, $p^{r-2} x \in \Lambda_{2}^{\max }$ holds for all isotropic $x \in \Lambda_{1}^{\min }$, and we obtain

$$
p^{r-1} \Lambda_{2}^{\max }=p^{r-1}\left\{z \in\left(\Lambda_{2}^{\max }\right)^{\#} \mid Q(z) \in R\right\} \subseteq p\left\{x \in \Lambda_{1}^{\min } \mid Q(x) \in p R\right\}^{\#} \subseteq \Lambda_{1}^{\min }
$$

by dualizing and applying Lemma 2.5. Moreover, if $k=n$ holds we have similarly

$$
p^{r-2} p\left(\Lambda_{1}^{(i)}\right)^{*} \subseteq \Lambda_{2}^{\max }, \quad p^{r-1} \Lambda_{2}^{\max } \subseteq \Lambda_{1}^{(i)} .
$$

If we have $p^{r-1} x \in \Lambda_{2}^{\min }$ the primitivity of $p^{r-1} x$ in $\Lambda_{2}^{\max }$ implies $b\left(p^{r-1} x, \Lambda_{2}^{(j)}\right)=$ $R, b\left(p^{r-1} x, \Lambda_{2}^{(j+1)}\right)=p R$ for some $0 \leq j<n$. We choose $y \in \Lambda_{2}^{(j)}$ isotropic with 
$b\left(p^{r-1} x, y\right)=1$ and have $p y$ primitive in $\Lambda_{2}^{(j+1)}, \ldots, \Lambda_{2}^{\min }$. The unimodular hyperbolic plane $R p^{r-1} x+R y$ then splits off orthogonally in $\Lambda_{2}^{\max }, \ldots, \Lambda_{2}^{(j)}$ and the $p$ modular hyperbolic plane $R p^{r-1} x+R p y$ splits off orthogonally in $\Lambda_{2}^{(j+1)}, \ldots, \Lambda_{2}^{\min }$. If on the other hand $p^{r-1} x \notin \Lambda_{2}^{\text {min }}$ holds there exists $0 \leq j<n$ with $p^{r-1} x \in$ $\Lambda_{2}^{(j)}, p^{r-1} x \notin \Lambda_{2}^{(j+1)}$. Since $p^{r} x$ is primitive in $\Lambda_{2}^{(j+1)}$ with $b\left(p^{r} x, \Lambda_{2}^{(j+1)}\right)=p R$, we have $p^{r} x \in p\left(\Lambda_{2}^{(j+1)}\right)^{*}$ primitive and find isotropic $y^{\prime} \in\left(\Lambda_{2}^{(j+1)}\right)^{*}$ with $b^{*}\left(p^{r-1} x, y^{\prime}\right)=$ 1. With $y=p y^{\prime} \in \Lambda_{2}^{\min }$ we have $b\left(p^{r} x, y\right)=p$ and $b\left(y, \Lambda_{2}^{(j+1)}\right)=p R$, and the $p$ modular hyperbolic plane $R p^{r} x+R y$ splits off orthogonally in $\Lambda_{2}^{(j+1)}, \ldots, \Lambda_{2}^{\min }$, whereas the unimodular hyperbolic plane $R p^{r-1} x+R y$ splits off orthogonally in $\Lambda_{2}^{\max }, \ldots, \Lambda_{2}^{(j)}$.

In both cases, by our assumptions we have $p^{r-1} y \in \Lambda_{1}^{\max }$. If in addition $p^{r-1} y \in$ $\Lambda_{1}^{\text {min }}$ holds, the unimodular hyperbolic plane $R x+R p^{r-1} y$ splits off orthogonally in $\Lambda_{1}^{\max }, \ldots, \Lambda_{1}^{(k)}$. If we had $b\left(p^{r-1} y, \Lambda_{1}^{(k+1)}\right)=R$ this would imply $k+1<n$ and the existence of a vector $x^{\prime} \in \Lambda_{1}^{(k+1)}$ with $b\left(p^{r-1} x^{\prime}, \Lambda_{2}^{\max }\right)=R$, which contradicts the maximality property of $k$. The $p$-modular hyperbolic plane $R p x+R p^{r-1} y$ therefore splits off orthogonally in $\Lambda_{1}^{(k+1)}, \ldots, \Lambda_{1}^{\min }$.

If $p^{r-1} y \notin \Lambda_{1}^{\text {min }}$ holds we have seen that we must have $k=n$ and hence $x \in$ $p\left(\Lambda_{1}^{(i+1)}\right)^{*} \subseteq \Lambda_{1}^{\min }$ and $b\left(x, \Lambda_{1}^{(i)}\right)=R, p^{r-1} \Lambda_{2}^{\max } \subseteq\left(\Lambda_{1}^{(i)}\right)$, hence $p^{r-1} y \in \Lambda_{1}^{(i)}$. From $b\left(x, p^{r-1} y\right)=1$ we see that $y \notin \Lambda_{1}^{(i+1)}$ so that $p^{r} y$ is primitive in $\Lambda_{1}^{(i+1)}, \ldots, \Lambda_{1}^{\min }$. The unimodular hyperbolic plane $R x+R p^{r-1} y$ then splits off orthogonally in $\Lambda_{1}^{\max }, \ldots, \Lambda_{1}^{(i)}$, whereas the $p$-modular hyperbolic plane $R x+R p^{r} y$ splits off orthogonally in $\Lambda_{1}^{(i+1)}, \ldots, \Lambda_{1}^{\mathrm{min}}$, and we can again reduce the Witt index $n$ by 1 and obtain the assertion from the induction hypothesis.

\section{REFERENCES}

[1] P. Abramenko, G. Nebe: Lattice chain models for affine buildings of classical type, Math. Ann. 322 (2002), no. 3, 537-562.

[2] F. Bruhat, F, J. Tits: Schémas en groupes et immeubles des groupes classiques sur un corps local. II. Groupes unitaires, Bull. Soc. Math. France 115 (1987), no. 2, 141-195.

[3] M. Eichler: Quadratische Formen und Orthogonale Gruppen, Grundlehren d. math. Wiss. 63, Springer Verlag 1952.

[4] W. Frisch: The cohomology of $S$-arithmetic spin groups and related Bruhat-Tits buildings, doctoral dissertation Göttingen 2002.

[5] P. Garrett: Buildings and Classical Groups, Chapman and Hall 1997.

[6] M. Kneser: Quadratische Formen, Springer Verlag 2002.

[7] I. Satake: Theory of spherical functions on reductive algebraic groups over $\mathfrak{p}$-adic fields. Inst. Hautes Études Sci. Publ. Math. 18 1963, 5-69.

Rainer Schulze-Pillot

Fachrichtung Mathematik

Universität des Saarlandes

Postfach 151150, 66041 Saarbrücken, Germany

email: schulzep@math.uni-sb.de 